\title{
Neuroética y deporte. ¿Una difícil relación?
}

\author{
Neuroethics and sport: A difficult relation?
}

\author{
FRANCISCO JAVIER LÓPEZ FRÍAS \\ Universidad de Valencia
}

Recibido: 15-1-2012

Aprobado definitivamente: 18-6-2012

\begin{abstract}
RESUMEN
Este trabajo analizará cuáles son las posibles consecuencias que los descubrimientos de la neurociencia pueden tener sobre la reflexión ética en torno al deporte. Siguiendo los trabajos en neuroética de Jonathan Haidt y Joshua Greene, parece ser que nuestro comportamiento moral está basado en una serie de mecanismos automáticos neuronales de carácter emotivo que acontecen en nuestros cerebros. Estos mecanismos podrían ser útiles a la hora de explicar el comportamiento de los hinchas deportivos, sin embargo, convertirían en inútiles los esfuerzos de la ética del deporte por establecer el principio del fair play como regulador ético de la práctica deportiva. Este trabajo ofrecerá dos contra-argumentos que mostrarán que, primero, nuestro comportamiento no está determinado por ciertos mecanismos automáticos que acontecen en el cerebro y, segundo, de hecho, la configuración de nuestro cerebro facilitaría en ciertos casos la posibilidad de que los hinchas desarrollen un comportamiento más ético. Por lo tanto, las metas de la ética del deporte no se ven socavadas completamente por los descubrimientos de las neurociencias.

\section{PALABRAS CLAVE \\ ÉTICA DEL DEPORTE, NEUROÉTICA, HINCHA DEPORTIVO, JUEGO LIMPIO}

\begin{abstract}
This work shall analyze whether or not the recent studies on neuroethics and neighbour disciplines have a critical impact on the ethical reflection on sports. Given Jonathan Haidt's and Joshua Greene's works on neuroethics, our moral behaviour seems to be mainly rooted in emotive mechanisms within our brain. If this is true, then sports supporters' behaviour can easily be explained by appealing to such emotive brain mechanisms. However, they will turn the efforts to apply the moral principle that is supposed to regulate the sporting world - the fair play principle-into useless efforts. I shall give two counter-arguments that will show, firstly, that supporters' misbehaviour cannot be totally explained by pointing out to several neural-natural-mechanisms, and, secondly, that our brains' make-up can, nevertheless, make sports supporters more willing to abide by the fair play principle. Therefore, sport ethics' aims are not undermined by neuroscientific knowledge.

KEYWORDS

SPORTS ETHICS, NEUROETHICS, SPORTS SUPPORTER, FAIR PLAY

PRINCIPLE
\end{abstract}




\section{NEUROÉTICA Y DEPORTE: EL SEGUIDOR DEPORTIVO Y SU CEREBRO EMOCIONAL}

MUCHO SE HA ESCRITO DENTRO DE LOS ÁMBITOS de la antropología y la sociología sobre el comportamiento de los seguidores deportivos -especialmente, respecto a aquellos grupos más violentos a los que nos referimos como hooligans. Sin embargo, existen muy pocos trabajos al respecto que provengan del campo de la ética. Entre la bibliografía de lo que podríamos denominar como «la ética del seguidor deportivo» los dos textos de referencia son: el artículo de Nicholas Dixon «The Ethics of Supporting Sports Teams» -que desde un punto de vista normativo trata de establecer cuál sería la tipología más virtuosa de seguidor o hincha deportivo. Y, en segundo lugar, el libro Your Brain on Cubs: Inside the Heads of Players and Fans editado por Dan Gordon.

Lo interesante de este último es que se trata de una recolección de artículos de autores que provienen de la neurociencia, la psicología moral evolutiva, o la ética. La mayoría de los artículos que componen esta colección tienen un carácter descriptivo, casi nunca normativo. Sin embargo, la línea que separa ambos terrenos es tan delgada que muchos acaban cruzándola, generando pretensiones normativas de aquello que, en principio, sólo poseía un carácter empírico-descriptivo. De este modo, la ética acaba naturalizada.

La naturalización de la ética es un suceso común siempre que ésta entabla una estrecha relación con la ciencia. Ya Spinoza, en la época de auge de la matemática, trató de elaborar una ética demostrada geométricamente. El darwinismo social y su influencia sobre la ética en el siglo XIX es otra ilustración de este fenómeno, así como también lo es la revitalización de este naturalismo que han provocado las disciplinas científicas más exitosas del S. Xx: la genética y, más recientemente, la neurociencia. Ya Hume trató de enfrentarse a tal problema al destapar la falacia naturalista consistente en la derivación de principios respectivos al debe de hechos empíricos concernientes al es. La ética kantiana y su enfrentamiento con las éticas materiales de raíz aristotélica, que pretendían derivar lo moral de contenidos empíricos, es otra de las posiciones filosóficas paradigmáticas referentes a la relación entre lo puramente empírico y los contenidos y forma de la moral.

Este fenómeno es fácilmente perceptible en la mayoría de los textos que componen el libro editado por Dan Gordon. En la mayoría de ellos parece existir un presupuesto de fondo común que afirma: dado que el cerebro de los espectadores les hace comportarse de un modo determinado, la configuración psico-biológica del mismo determina la forma y el contenido de su comportamiento moral -es decir, el «es» acaba llevando al «debe». Éste es, por ejemplo, el caso del artículo de Bennet Foody titulado «Risk and Asterisk», que tras analizar empíricamente los mecanismos neuronales que están tras la conexión que se establece entre el ritmo del evento deportivo y sus seguidores, afirma que deberían tomarse «medidas psico-biológicas» para que los espectadores estén más metidos en y colaboren más con la tensión propia de dichos eventos. Desde una posición naturalista, asegura que dichas medidas deben consistir en mejorar neuronalmente a los espectadores deportivos -por ejemplo, a través de la ingestión de cerveza- para que optimicen los mecanismos cerebrales que les permiten disfrutar del juego:

La mejora neuronal activa los neurotransmisores inhibitorios GABA del cerebro e inhibe los neurotransmisores excitatorios glutamato. Ésta también provoca la segregación de dopamina y 
serotonina en los centros de recompensa del cerebro. Como consecuencia, produce sentimientos de bienestar y euforia y alienta la participación social en el seguimiento del juego. ${ }^{1}$

Como más tarde analizaré en detalle, éste es el mismo error naturalista que cometen los famosos trabajos de Joshua Greene y Jonathan Haidt. Quiénes, aunque afirman estar realizando sólo una descripción de lo que acontece en nuestros cerebros, sin embargo, acaban concluyendo que aquello que sucede hemos de aceptarlo como determinante de la forma y el contenido de la moral. Mientras este naturalismo ético se extiende en el ámbito académico -ya se habla de neuroética, así como de neuropolítica, neuroeconomía, neuroreligión, y otros muchos ámbitos con la raíz «neuro-»-, este artículo analiza las posibles consecuencias que ello puede tener para una disciplina como la ética del deporte.

Si hay un concepto central con el que debe enfrentarse cualquiera que trabaje dentro del ámbito de la ética del deporte, ése es el de fair play. Éste es el ideal regulativo de la práctica deportiva. Aunque comenzó siéndolo sólo para los propios deportistas -los principales protagonistas-, actualmente ya se utiliza en términos mucho más amplios con el fin de que éste alcance a todos los elementos esenciales de la práctica deportiva. Uno de ellos es, por supuesto, la comunidad conformada por sus seguidores. Por lo tanto, en este artículo se analizará si, dados los recientes estudios en neurociencias y sus implicaciones para la ética, sigue teniendo sentido para la ética del deporte luchar por la inculcación del espíritu del fair play en los hinchas.

\section{NEUROÉTICA Y EL COMPORTAMIENTO DEL HINCHA DEPORTIVO}

\section{II.1. Los experimentos de Joshua Greene: la ligazón al cercano}

En el famoso artículo de J. Greene titulado «From neural «is» to moral «ought»: what are the moral implications of neuroscientific moral psychology?» éste nos propone dos dilemas morales diversos:

a) Vamos conduciendo por una carretera $y$, de pronto, advertimos a un excursionista herido cuyas piernas están sangrando. Si no le llevamos al hospital en nuestro coche, el excursionista corre altos riegos de morir o, cuanto menos, perder las piernas. Llevar a cabo tal acción supone arruinar las nuevas tapicería de cuero del coche, que costaron $200 €$.

b) Recibimos una carta de una reconocida ONG. En ella se afirma que con una simple donación de $200 €$ se salvarán bastantes vidas en un país en vías de desarrollo.

J. Greene afirma que, mientras que sí consideraríamos como un monstruo moral a aquel que negara la ayuda al excursionista, no haríamos lo mismo con aquel que hiciera caso omiso a la carta de la ONG. Sin embargo, ambas tienen el mismo efecto: salvar vidas. ¿Por qué se da esta disparidad de juicio? Joshua Greene trata de explicarlo distinguiendo entre dilemas morales personales e impersonales. Los primeros son aquellos en los que aquel que sufre las consecuencias de nuestra acción está a la vista, frente a nosotros. Mientras que los segundos son aquellos casos en los que, al contrario, los sujetos son lejanos y no tenemos percepción alguna de ellos. A través de técnicas de escaneo cerebral, J. Greene descubrió que los dilemas morales personales provocaban

\footnotetext{
${ }^{1}$ Foody, B. «Risk and Asterisks: Neurological Enhancements in Baseball» en Gordon, D., Your Brains on Cubs: Inside the Heads of Players and Fans, Danna Press, New York, 2007, p. 95.
} 
una mayor activación de las áreas cerebrales asociadas con la emoción y la cognición social. De modo que, al parecer, lo que consideramos como la acción moral, va ligado, en principio, a ciertas reacciones emotivas y automáticas que acontecen dentro de nuestro cerebro.

Los experimentos de Jonathan Haidt al respecto y, sobre todo, el hallazgo por parte de Benjamin Libet de un impulso cerebral, denominado por él como «potencial de disposición», que acontece en nuestro cerebro de forma que precede en unas milésimas de segundo a la percepción consciente de nuestros actos, han provocado la aparición de una corriente mayoritaria dentro de las neurociencias que defiende que los juicios morales no son más que racionalizaciones post hoc de ciertos impulsos emotivos que acontecen en nuestro cerebro y que, inmediatamente, nos están determinando a afirmar si algo es correcto moralmente o no lo es. De modo que la base de nuestros juicios morales es, eminentemente, emotiva y natural. En palabras de J. Haidt, «nuestras emociones e intuiciones dirigen nuestro razonamiento moral, tan seguro como un perro que agita su cola». ${ }^{2}$ A lo que Green añade "gente necesitada que es «cercana y personal» acciona nuestro botones emocionales, mientras que aquellos que están lejos de nuestro campo de visión queda fuera de nuestra mente». ${ }^{3}$

El hecho importante que muestran estos trabajos en relación a la cuestión que analiza este artículo -el comportamiento moral de las hinchas deportivos- es que tanto J. Greene como J. Haidt, sitúan el origen de esta predisposición cerebral de los sujetos a preocuparse por y considerar moralmente al cercano -a la vez que obviamos al lejanoen la naturaleza gregaria y comunitaria de nuestros ancestros; nuestro juicio moral evolucionó de manera que nuestro instinto altruista o empático sirviera para salvaguardar la integridad de los sujetos a través de la mantención y protección de las pequeñas comunidades de las que formaban parte.

Sólo con aquellos que tenemos un contacto cercano y personal desarrollaremos actitudes que podemos considerar como verdaderamente morales, pues era sacrificándose por los otros miembros de su propia comunidad cómo nuestros ancestros podían, a su vez, salvaguardar su integridad. Este espíritu de colmena quedó grabado en nuestra mente y evolucionó más allá de la mera preocupación por la integridad del yo para llevarnos a proteger a aquellos que forman parte de nuestra comunidad, a la vez que a rechazar a aquellos que compiten contra ellas y las ponen en peligro. J. Haidt en su recientemente publicado The Righteous Mind: Why Good People are Divided by Politics and Religion denomina esos procesos neuronales relacionados con la superación del egoísmo individualista el «interruptor de la colmena» (the hive switch). ${ }^{4}$

Sabemos que los sentimientos de grupo, conexión y pertenencia que existen entre los hinchas deportivos son de los más fuertes de entre las comunidades actuales. Estos grupos parecen ser sólo comparables a los partidarios de una religión o un grupo político. Es más, Jonathan Haidt en su último libro, anteriormente citado, en su tarea de tratar de analizar las bases neuronales del partidismo político y la devoción religiosa, lleva a cabo una gran cantidad de referencias y analogías que relacionan el deporte con las actividades religiosas y las políticas. Por ejemplo, hablando del patriotismo americano, afirma que los atentados del 11 de septiembre accionaron el interruptor

\footnotetext{
${ }^{2}$ Haidt, J. «The emotional dog and its rational tail», p. 830 en: Psychological Review, 2001, Vol. 108 (4), pp. 814-834.

${ }^{3}$ Greene, J. «From neural «is» to moral «ought»: what are the moral implications of neuroethics moral psychology?» p. 849 en Nature Review. Neurosciences, Oct., 2003, Vol. 4 (10), pp. 846-849.

${ }^{4}$ Haidt, J. The Righteous Mind: Why Good People are Divided by Politics and Religion, London, Allen Lane, 2012.
} 
emotivo que hizo a los americanos «apoyar a sus equipos como nunca los habían hecho antes» [p.190]. Además, el capítulo 11 se titula «La religión es un equipo deportivo».

Matteo Forgiarini, Marcello Galluci y Angelo Maravita han llevado a cabo unos experimentos que sustentan la tesis de que ciertos niveles de empatía sólo son alcanzables entre los sujetos que comparten ciertos rasgos comunes. ${ }^{5}$ Concretamente, los experimentos son dos: el primero se realizó a través de la medición de la respuesta galvánica o conductancia de la piel (SRC), el segundo por medio del Test de asociación implícita.

1) Se proyectaron 12 vídeos protagonizados por 6 actores; dos africanos, dos caucásicos y dos asiáticos. Cada uno de ellos protagonizaba dos vídeos; en uno de ellos el actor sufría una acción dañina y en el otro una inocua. Los registros de la conductancia de la piel mostraron que los individuos experimentaban una reacción mayor en las escenas de daño y, además, la reacción era mayor cuando el individuo coincidía en raza con el sujeto del experimento.

2) El segundo experimento conservó el mismo paradigma, es decir, identificar la reacción empática que el sujeto siente ante seres de diversas razas cuando éstos sufren algún daño. En este caso, lo determinante no fue la conductancia de la piel, sino el tiempo de reacción de los participantes en una práctica llevada a cabo por ordenador. En ella, aparecían dos columnas: una con una foto de una persona africana o caucásica sufriendo una acción, y otra con conceptos positivos -amor, paz, cariño- o negativos dolor, sufrimiento, guerra. El experimento, llevado a cabo entre caucásicos, mostró que éstos establecían dos tipos de relaciones de un modo más rápido que el resto: el actor caucásico con un concepto positivo y el actor africano con el concepto negativo.

A juicio de M. Forgiarini esto se debe a que «cuánto más cercano es un rasgo fenotípico entre el que actúa y el observador, más fuerte es la respuesta empática psicofisiológica hacia él». ${ }^{6}$ Es cierto que algunos rasgos fenotípicos son más relevantes que otros, por ejemplo, la raza parece psico-fisiológicamente más relevante que, por ejemplo, la pertenencia a un club. Sin embargo, afirman nuestros autores, cualquier forma de pertenencia grupal «puede tener un papel importante en la generación de empatía», ${ }^{7}$ ya que "ser consciente del ambiente cálido que existe dentro de grupos externos y la competencia afecta a las emociones que los grupos externos provocan en el sujeto». ${ }^{8}$ A juicio de estos autores, estos datos también poseen una explicación evolutiva, ya que «la pertenencia a un grupo racial define el tipo de colación y alianza producidas a lo largo de la evolución y, además, produce una importante modulación de los sustratos neuronales de los componente emocionales de la empatía». ${ }^{9}$

Como consecuencia de estos experimentos, puede decirse que cuando J. Haidt o J. Greene utilizan la expresión «a la vista», no lo hacen de forma que todo aquel que está presente físicamente, a la vista, recibe consideración moral, sino todo aquel que está «a la vista» emotivamente hablando. La pertenencia a un mismo club o a un club amigo puede ser suficiente para activar dichos mecanismos de generación de empatía, mientras que seguir a un equipo rival puede hacer que los rivales queden respectivamente fuera de la vista moral de los otros. Estos son los mecanismos que

\footnotetext{
${ }^{5}$ Forgiarini, M.; Galluci, M; y Maravita, A. «Racism and the Empathy for Pain on Our Skin» en: Frontiers in Psychology, 2011, Vol. 2, p.108.

${ }^{6}$ Ibíd.

${ }^{7}$ Ibíd.

${ }^{8}$ Ibíd.

${ }^{9}$ Ibíd. p. 5.
} 
están a la base del comportamiento grupal de las comunidades humanas, sin embargo, en algunas de ellas los lazos emotivos se engrandecen y provocan mayores niveles de adhesión, casos claros de ello son la política, la religión y el deporte. En el siguiente apartado trataré de analizar los procesos neuronales que pueden explicar tales diferencias.

\section{II.2. Hábitos e identidades establecidos: desde el sistema de recompensa al emocional}

Tanto el deporte, como la política y la religión, parecen ser, en palabras de Jonathan Haidt, un tipo de actividades que provocan que los sujetos vivan en realidades tan distintas y, a veces, opuestas, que el diálogo y el trato cordial y afectivo entre ellas es imposible. ${ }^{10}$ Éste es el presupuesto que me interesa analizar para comprobar si los hinchas deportivos están o no tan determinados por estas conexiones neuronales, que exigirles que se comporten moralmente -o «de forma limpia» en términos deportivosparece algo imposible. Si recordamos la cita con la que se abre este artículo, entonces, esos «discutir, duda, argumentar y mantener la menta abierta» por los que aboga su autor parecerían casi imposibles. E.G. Marcus, analizando la formación de los hábitos e identidades políticas de los ciudadanos, ofrece una explicación neuronal de este fenómeno, la cual explicitaré a continuación.

Como explica Patricia Churchland, ${ }^{11}$ las habilidades sociales son adquiridas y los hábitos son formados en base a experiencias emotivas de dolor y satisfacción que afectan a nuestro sistema de recompensa neurológico. Remarcando la importancia de las emociones para la formación de nuestros hábitos, E.G. Marcus trata de analizar el partidismo político y los hábitos políticos de los individuos apelando, precisamente, a los sistemas emotivos del cerebro. ${ }^{12}$ Según este autor, nuestro cerebro posee tres sistemas de emoción diferentes: el sistema de lucha y huída, el sistema de disposición y el sistema de vigilancia. El más estudiado de éstos es el sistema de lucha y huída que, situado en el tálamo, está diseñado para proteger al individuo de la amenaza inminente e innata. Sin embargo, los dos sistemas más directamente implicados en el comportamiento político de los individuos son: el sistema de disposición, que está implicado en el aprendizaje y la ejecución de hábitos, y el sistema de vigilancia.

El sistema de disposición posee la importante tarea de proveer información sobre el éxito o fracaso de una acción que está en marcha. Para ello, utiliza una serie de marcadores emocionales que señalan el éxito o el fracaso en cada momento de realización de la tarea. Sin embargo, mientras el cerebro está centrado en una tarea, necesita un segundo sistema defensivo cuya función es la de mostrar cuándo las circunstancias dadas son lo suficientemente seguras como para actuar. Éste es el papel del sistema de vigilancia, el cual monitoriza dos niveles de información: 1) el actual plan de acción y 2) las corrientes sensoriales en uso. Las amenazas e intrusiones son señaladas por medio de estados de ánimo de pronunciados: bien sea la ansiedad ${ }^{13}$ cuando las cosas no marchan según lo esperado- o bien el entusiasmo -cuando todo está marchando correctamente. ${ }^{14}$

\footnotetext{
${ }^{10}$ Haidt, J. op. cit. (2012), p. 231.

11 Churchland, P. Braintrust: what neuroscience tells us about morality, Princenton, Princenton University Press, 2011, p. 165.

${ }^{12}$ Marcus, E.G. The Sentimental Citizen: emotions and democratic politics, University Park (PA), Pennsylvania State University Press, 2002,

${ }^{13}$ Ibíd., p. 74.

${ }^{14}$ Ibíd p. 80.
} 
Ansiedad y entusiasmo son, por lo tanto, las herramientas emocionales que utiliza nuestro cerebro para diferenciar lo exitoso de aquello que no lo es. Por lo tanto, los hábitos que aprendemos son aquellos que eliminan la ansiedad de nuestras vidas y, por el contrario, nos permiten alcanzar estados de bienestar y entusiasmo a través de las tareas que llevamos a cabo. Naturalmente, estos hábitos nos ligan a aquellos grupos sociales, clanes, comunidades, etc., en los que ellos son posibles. Cuando el ser miembro de cierta comunidad y el placer que reporta la pertenencia a ella se retroalimentan positivamente, entonces surge un problema: el entusiasmo nos hace centrarnos tan atentamente en una tarea que perdemos la capacidad de adoptar múltiples perspectivas, es decir, el entusiasmo inhibe el criticismo, la duda y la aceptación de lo diverso. En palabras de E. G. Marcus:

Los hábitos siempre serán vigorosamente defendidos podamos o no justificarlos en términos de principios, porque una vez que forman parte de nuestro repertorio de habilidades, proporcionan por sí mismos los medios suficientes para asegurarnos alcanzar las metas de nuestra vida [...] Dejar un hábito es abrir un vacío práctico. ${ }^{15}$

Tras todo lo considerado, coincido con la idea expuesta por Patricia Churchland que señala que parece existir una especie de imperativo neuronal que promueve la sociabilidad y la asociación voluntaria entre los individuos con el fin de asegurar su propio bienestar ${ }^{16} \mathrm{y}$ auto-conservación. Aunque es cierto que, dada la naturaleza cambiante de las sociedades humanas, nuestros cerebros se han adaptado a formas más amplias de sociabilidad -expandiendo el sentido innato de cuidar de la descendencia hacia los cercanos, los amigos e, incluso, los extraños ${ }^{17}$ - el «espíritu de la colmena» heredado de nuestros ancestros parece permanecer intacto. Por lo tanto, a la luz de dicho imperativo neuronal, tal cosa como el juicio crítico es imposible para el espectador deportivo, ya que considera todo aquello que pone en peligro las comunidades en que desarrolla su vida de una forma placentera como una competencia que puede poner en peligro, precisamente, ese bienestar que le reporta el pertenecer a ciertas comunidades. El rival no está a la vista moralmente, pues es un obstáculo a superar, es el enemigo.

Un reciente estudio realizado en la pasada final del Mundial de Fútbol de Sudáfrica entre España y Holanda, llevado a cabo por el Laboratorio de Neurociencia Social de la Universidad de Valencia, ${ }^{18}$ ha mostrado que durante aquel partido los niveles de testosterona y de cortisol de los individuos analizados aumentaban significativamente durante el encuentro, pero no tras él. A juicio de estos datos, los investigadores afirmaron que el sistema nervioso concibió la posibilidad de que su equipo perdiera el partido como una amenaza para su identidad social, de ahí el incremento de los niveles de cortisol, que es una hormona relacionada con el estrés y la ansiedad. Dado que los individuos sintieron su yo social amenazado, los niveles de testosterona, hormona relacionada con la competición social, también se elevaron haciendo más propensos a los sujetos a ver a los rivales como una amenaza y como un fiel compañero a aquel que pertenecía a la misma comunidad. Por ello, es por lo que J. Haidt afirma que la acción de estas hormonas «nos liga y nos ciega [...] sería genial

\footnotetext{
${ }^{15}$ Ibíd., p.92.

${ }^{16}$ Churchland, P. op. cit. (2011), p. 27. «todo el sistema nervioso está organizado para hacerse cargo de la supervivencia del cuerpo del que es parte»; Cortina, A. Neuroética y Neuropolítica. Las bases para la educación moral, Madrid, Tecnos, 2011.

${ }^{17}$ Churchland, P. op. cit. (2011), p. 46.

${ }^{18}$ Corral, M. G. «El chute hormonal en los hinchas del fútbol» en El Mundo, 19-04-2012.
} 
creer que los humanos estamos diseñados para amar a todos de modo incondicional. Genial, pero improbable desde un punto de vista evolutivo». ${ }^{19}$

\section{III. ¿ENCAJA EL IDEAL DEL FAIR PLAY CON LA CONFIGURACIÓN NEURAL DE LOS HINCHAS? UNA NEUROÉTICA DEL DEPORTE}

\section{III.1. Fair play y nuestra configuración emocional}

En el clásico libro de Robert L. Simon titulado Fair Play podemos leer la siguiente afirmación sobre los seguidores deportivos:

Un requisito para una buena deportividad entre hinchas es que ellos mantengan su perspectiva crítica [...] con el fin de apreciar quién se ha enfrentado mejor al desafío. De no ser así, el deporte es reducido a un simple medio para satisfacer nuestros propios egos en vez de constituir un área donde espectadores y atletas al mismo tiempo pueden aprender y crecer entendiendo y enfrentando cada creciente desafío hacia su capacidad atlética y crítica. ${ }^{20}$

La pasada sección acabó señalando el hecho de que los hinchas parecen estar neuronalmente configurados a no reconocer como iguales a aquellos seguidores que son diferentes de ellos por el hecho de ser hinchas del equipo rival. Por lo tanto, ese espíritu crítico reclamado por Robert L. Simon parece, a primera vista, imposible, pues todo lo relacionado con los equipos y las comunidades rivales constituye una amenaza al bienestar y a la unión de la comunidad emotiva que cada hinchada conforma. Entonces ¿debe la ética del deporte aceptar que, dada nuestra configuración neuronal, la realización del principio del fair play no es más que una utopía que nunca alcanzaremos? Al menos eso es lo que se sigue de los análisis anteriormente expuestos. De ser así, aquel concepto con el que todo filósofo que se dedique a analizar el deporte debe tratar, a saber, el fair play, queda como un concepto éticamente inservible recordemos que el fair play es el idea regulativo que se aplica en todos los análisis éticos del fenómeno deportivo, sea la situación económica, las competición entre atletas o el comportamiento de los hinchas.

Con el fin de responder satisfactoriamente a aquella cuestión y salvar el estatus de la ética del deporte en el reto que la neurociencia le plantea: primero, aclararé el significado del término «fair play». Por cuestiones prácticas tomaré la noción aportada por Robert L. Simon como la correcta. ${ }^{21}$ A su juicio, la idea de fair play debe sustentarse en la noción de persona. De este modo, el rival nunca debe ser visto como un mero obstáculo que tiene que superarse a toda costa, sino como una persona cuya actividad reclama una respuesta apropiada. Por lo tanto, una competición regida atendiendo al principio del fair play presupone el igual respeto por el rival, quien no es un medio para conseguir un fin, sino un fin en sí mismo cuya especifidad debe ser reconocida. Como vemos, esta ideal no sólo se aplica al comportamiento del deportista, sino también al de los seguidores y, por supuesto, de las instituciones que también son partes esenciales del deporte.

\footnotetext{
${ }^{19}$ Haidt, J. op. cit. (2012), p. 191 y 245.

${ }^{20}$ Simon, R.L. Fair Play: sport, values and society, Oxford, Westview, 1991, p. 111-112.

${ }^{21}$ Llevar a cabo un análisis exhaustivo del concepto de fair play, así como, de cuál es mi concepción específica al respecto, primero, conllevaría desviar la discusión que está en marcha, y, segundo, requeriría un espacio del que no se dispone en este trabajo.
} 
Centrándonos en la cuestión del comportamiento de los hinchas. Parece claro que este ideal es irrealizable desde el mismo momento en que los hinchas se conciben a sí mismos como miembros de un grupo que inevitablemente se opone a los grupos rivales; los cuales no son reconocidos como sujetos dignos de consideración moral -ya que no accionan el interruptor emotivo de nuestros cerebros-, sino como obstáculos o peligros que impiden que el propio grupo «satisfaga su ego» -tomando los términos de Robert Simon. En lo que sigue expondré dos contraargumentos a esta idea de la imposibilidad del ideal del fair play respecto al comportamiento de los seguidores deportivos. El primero está basado en la posición kantiana respecto a la neuroética desarrollada por la filósofa española Adela Cortina, mientras que el segundo se basa en ideas propias del ámbito de las neurociencias, concretamente la neuroeconomía y la neuroética.

\section{III.2. La falacia naturalista, hermenéutica y moral}

La cuestión a responder en este apartado es si se pueden o no establecer pretensiones normativas a través de, simplemente, señalar la correlación empírica entre el cerebro y el comportamiento moral. Parece claro que las ciencias tratan de esclarecer aquello que las cosas son, mientras la moral trata de alcanzar aquello que deberíamos hacer. Ésta es la distinción que Kant desarrolló con el fin fundamentar racionalmente la moral. Así, el de Königsberg afirma en La fundamentación de la metafísica de las costumbres:

Cuando se trata del valor moral no importan las acciones, que se ven, sino aquellos íntimos principios de las mismas, que no se ven [...] no se trata aquí de si sucede esto o aquello, sino que la razón, por sí misma e independientemente de todo fenómeno, ordena lo que debe suceder. ${ }^{22}$

Lo que Kant está tratando de evitar en su Fundamentación es aquello que David Hume había formulado como la «falacia naturalista», que consiste en la negación de la posibilidad de derivar una afirmación de deber de una sobre el ser. Sin embargo, esto no significa que no podamos inferir o establecer principios de acción a partir de datos de la experiencia, de hecho, esto es lo que hacemos constantemente. ${ }^{23}$ Siguiendo las ideas de Hume, Patricia Churchland afirma que:

[...] un entendimiento más profundo de qué es aquello que hace a los humanos y a otros animales sociales, y qué es aquello que nos posibilita tener cuidado de los otros, puede conducir a un mejor entendimiento de cómo lidiar con problemas sociales. ${ }^{24}$

Aclara P. Churchland que los mecanismos neuronales de nuestro comportamiento social son sólo una plataforma -incluso son sólo un nivel de la plataforma-, no la historia completa de los valores morales humanos. ${ }^{25}$ Por lo tanto, con señalar simplemente algunas correlaciones causales entre la moral y los mecanismos neuronales no podemos establecer pretensiones de normatividad. Es necesario diferenciar entre tareas normativas y descriptivas de la ética, es decir, entre ética descriptiva y ética normativa. Las ciencias pueden ser muy útiles para el primer caso, sin embargo, no lo son en el segundo porque ellas no pueden decirnos cómo hemos de

\footnotetext{
${ }^{22}$ Kant I. Fundamentación de la metafísica de las costumbres, trad. de Adela Cortina y Jesús Conill, Madrid, Tecnos, 2005, pp. 38-39.

${ }^{23}$ Churchland, P. op. cit. (2011), p.5.

${ }^{24}$ Ibíd p. 4

${ }^{25}$ Ibíd.
} 
vivir. Aquello que sea lo bueno es demasiado complejo para ser reducido a una explicación científica mecánica de la realidad.

Con el fin de fundamentar esta idea, Patricia Churchland establece una acertada analogía entre lo bueno y lo sano. ${ }^{26}$ Ella señala que una simple fórmula no es suficiente para explicar la naturaleza de la salud y el bien ya que lo sano no puede ser igualado, por ejemplo, a tener la presión sanguínea baja, sino que existen otros elementos diversos que nos permiten poseer una vida sana, hay tantos que es imposible llegar a un acuerdo sobre la importancia o validez de todos ellos, por ejemplo, no todos los médicos afirman la necesidad de ejercicio físico. Al igual que el ámbito de la salud, el del comportamiento humano es demasiado complejo y amplio como para reducirlo a «fórmulas neuronales» que explican nuestro comportamiento apelando únicamente a cómo se configura y funciona nuestro cerebro, tal y como J. Greene y J. Haidt trataron de hacer.

Aunque tanto J. Greene como J. Haidt afirman en sus artículos que tienen en cuenta la objeción presentada por la falacia naturalista -por ello aseguran restringir su tarea al ámbito de la ética descriptiva tratando de evitar caer en el terreno de lo normativo o prescriptivo-, parece que las consecuencias de sus argumentos sobrepasan lo descriptivo ya que están reclamando que el modo en que nuestro cerebro funciona determina inevitablemente nuestro comportamiento moral. A su entender, no podemos deshacernos de nuestra configuración neuronal, por ello, J. Greene reclama que tendríamos que aceptarla y cambiar nuestro modo de auto-concebirnos moralmente con el fin de lograr sociedades mejores a las actuales:

Un mundo lleno de gente que considera sus convicciones morales como reflejo de sus valores personales en vez de cómo reflejo de una «verdad moral objetiva» podría ser un lugar más feliz y pacífico que el mundo que actualmente habitamos. ${ }^{27}$

De acuerdo con la analogía establecida por Patricia Churchland, este conocimiento científico puede mostrarnos las condiciones que contribuyen a tener una vida más sana o moral con el fin de facilitarnos la elaboración de teorías normativas más factibles -que encajen mejor con nuestra naturaleza. Sin embargo, la ciencia no puede resolver dilemas morales:

Hemos de estar abiertos a la posibilidad de que un conocimiento más profundo de la naturaleza de nuestra sociabilidad puede arrojar luz sobre algunas de nuestras prácticas e instituciones, y hacernos pensar de modo más sabio sobre ellas. ${ }^{28}$

En la misma línea, pero siguiendo el pensamiento kantiano de Jürgen Habermas, la filósofa Adela Cortina señala que el ser humano ha desarrollado dos tipos de lenguaje totalmente diversos a lo largo de su evolución histórica, por un lado, el lenguaje del observador cuyo objetivo es alcanzar un conocimiento objetivo y válido universalmente $\mathrm{y}$, por otro, el del participante que busca alcanzar acuerdos intersubjetivos entre los diversos participantes. ${ }^{29}$ Este segundo lenguaje es la base del pensamiento moral; su naturaleza no puede configurarse en términos de causales, sino en términos de condiciones que influyen en nuestro autorreconocimiento como individuos responsables e iguales. A juicio de J. Habermas y de A. Cortina, el reconocimiento mutuo intersubjetivo de que todos nosotros poseemos una conciencia libre y responsable es

\footnotetext{
${ }^{26}$ Ibíd p.198.

${ }^{27}$ Greene, J. op. cit. (2003), p. 847.

${ }^{28}$ Churchland, P. op.cit. (2011), p. 190.

${ }^{29}$ Habermas J. Entre naturalismo y religión, Barcelona, Paidós, 2006.
} 
suficiente para hacerla posible. Es cierto que puede haber ciertos inputs y outputs que pueden influir en nuestro modo de pensar, pero no son más que condiciones que influyen en nuestros procesos intelectivos, no son causas directas de ellos. ${ }^{30}$

Complementando esta distinción habermasiana con la distinción leibniziana entre razones necesarias y razones suficientes, Adela Cortina afirma que debemos distinguir entre «bases» y «fundamentos» con el fin de clarificar las relaciones que se establecen entre las ciencias y la ética. ${ }^{31}$ Cuando afirmamos que algo es la base de otra cosa, nos estamos refiriendo a que es algo necesario para explicarlo, pero ello no implica que sea suficiente. Sin embargo, si decimos que algo es el fundamento de otra cosa, su sola presencia conduce al efecto, es decir, a aquello que deseamos explicar. Esta idea está completamente relacionada con la afirmación de Patricia Churchland de que nuestros mecanismos neuronales no son más que una plataforma de la moralidad bases- y no la historia completa -fundamento. Nuestra configuración neuronal es una de las bases que condiciona nuestro comportamiento, pero también hay bases sociales, biológicas y demás.

Aplicando esta crítica a las pretensiones de la neurociencia y su relación con la ética al tema que nos ocupa en este trabajo. Podría decirse que bien es cierto que los seguidores deportivos de un equipo o deportista, bien pueden sentir de modo instantáneo ciertas emociones negativas, repulsivas e incluso de odio hacia los rivales. $\mathrm{Y}$ que, por el contrario, los miembros de una misma comunidad de seguidores se perciban como más cercanos, más merecedores de respeto y consideración. Sin embargo, esto no deja de ser una de las bases que determina el comportamiento del hincha. Otras consideraciones y factores también entran en juego, es más, esas reacciones emotivas en el cerebro podrían modificarse si las relaciones que se establecen entre hinchas fueran diversas a las que actualmente existen -en esta dirección va el segundo contra-argumento que presentaré. De este modo, no podemos afirmar que los recientes hallazgos en neuroética obliguen a la ética del deporte a cruzarse de brazos y concebir el ideal del fair play como irrealizable.

\section{III.3. El argumento neuronal. Nuestra configuración neuronal universalista}

En el caso de que algunos defensores de las neurociencias no queden convencidos por este argumento filosófico y aún piensen, siguiendo los experimentos de J. Greene y J. Haidt, que los hinchas deportivos están neuronalmente determinados a estar encerrados en el interior de las fronteras determinadas por la moral social de la hinchada a la que pertenece, trataré de presentar un argumento neuronal que defiende que: aunque estuviésemos determinados por nuestra configuración neuronal -es decir, que ésta no sólo fuera una base de la que depende nuestro comportamiento moral, sino su fundamento- a comportarnos como lo hacemos, la meta de realizar el ideal del fair play es aún posible porque nuestros cerebros poseen ciertos mecanismos de comportamiento universal que nos permiten reconocer la especifidad moral de cada ser humano más allá de su proximidad o identidad grupal. ${ }^{32}$

Tal y como se ha señalado anteriormente, siguiendo las afirmaciones de J. Green y J. Haidt, algunos autores dentro de la neuroética afirman que existe un imperativo biológico-neuronal que nos hace reconocer moralmente sólo a aquellos que son cercanos a nosotros en nuestras vidas diarias, es decir, a aquellos con los que

\footnotetext{
${ }^{30}$ Cortina, A. op. cit. (2011), p. 195-198.

${ }^{31}$ Ibíd

${ }^{32}$ Pfaff, D. The Neurosciences of Fair Play, New York, Danna Press, 2007.
} 
compartimos algún tipo de identidad grupal. Adela Cortina llama a este hecho «reciprocidad fuerte». Sin embargo, como Donald Pfaff señala, podemos comprobar que toda cultura existente ha desarrollado alguna versión de aquello que llamamos la Regla de Oro -que puede ser formulada como «haz según quieras que te hagan a ti»que debe ser aplicada no sólo a la gente con la que estamos en contacto de algún modo o con la que compartimos alguna identidad grupal, sino a todo ser humano existente, es decir, nos lleva a desarrollar una reciprocidad universal. Por ejemplo, esta reciprocidad universal es lo que Kant intentó captar al formular su imperativo categórico y también aquello que J. Habermas y K.O. Apel trataron de conseguir al apelar al presupuesto contrafáctico de la situación ideal de habla. ¿Cuál sería el correlato empírico-neuronal de este tipo de propuestas morales?

Donald Pfaff apela a dos procesos dentro de nuestro cerebro que, de alguna manera, ya son familiares para nosotros, las acciones hormonales que potencian y explican el comportamiento de cuidado hacia los otros y, en segundo lugar, las fuerzas que provocan nuestro comportamiento agresivo. Ambos le llevarán, al igual que hace E.G. Marcus, a considerar el rol del entusiasmo y la ansiedad. En su opinión, la posibilidad de que podamos desarrollar un comportamiento ético universal depende del balance entre ambas fuerzas. El amor y el miedo no sólo tienen la habilidad de hacernos comportarnos de un modo comunitario o, parafraseando a J. Haidt, de «conducirnos a la colmena», sino que pueden llevar a la disolución de las fronteras entre el «yo» y el «tu», por lo tanto, no es que los mecanismos neuronales estén en contra de la imparcialidad, sino que pueden posibilitarla con la acción de estas dos fuerzas.

Para comenzar, consideremos este ejemplo con cuatro pasos, que tomamos de Pfaff:

1) Una persona (A) considera llevar a cabo cierta acción con respecto a otra (B). Por ejemplo, un hincha del Real Madrid está considerando reírse de varios hinchas de Osasuna porque su equipo está siendo derrotado 8-1. El hincha del Real Madrid representa su acción en su córtex cerebral con el fin de considerar las relaciones de la acción y sus consecuencias, para ello,

2) (A) visualiza su objetivo, que es (B). Las neuronas de reconocimiento facial entran en juego. Éstas se encuentran en el córtex infratemporal y son responsables tanto del reconocimiento facial como de la actividad neuronal que produce el sentido del yo. También las neuronas espejo pueden tomar parte en este proceso. Por lo tanto, (A) activa las mismas zonas cerebrales que (B) con el fin de entender y reconocer sus intenciones. Cuando (A) hace eso, siente el dolor y la ansiedad que (B) está sufriendo, por lo tanto:

3) (A) borra su diferencia identitaria con (B) porque ha sufrido una sobreexcitación del cerebro de tal magnitud-sobre todo en la amígdala y el córtex prefrontal—que las neuronas encargadas de señalar la identidad propia borran nuestra diferencia con la conciencia del otro. Así, miedo.

4) es menos plausible que (A) ataque a (B) porque están compartiendo el mismo

Según Donald Pfaff, existe una especie de interruptor emocional en nuestros cerebros que:

cuando es accionado en una posición, «desconecta» la distinción yo-otro y las imágenes de ambos individuos [...] acaban fusionadas. El resultado es el comportamiento empático [...] 
Cuando el botón es accionado en la posición diferente, las imágenes permanecen estables para cada uno, entonces podrían herirse. ${ }^{33}$

Por lo tanto, el hecho de sentir la misma ansiedad y sufrimiento que los otros, hace a la gente considerar la no realización de una acción y pensar sobre su adecuación o corrección moral. Así que, aunque es cierto que normalmente nos comportamos siguiendo nuestros hábitos y costumbres, no es menos seguro que cuando emergen problemas morales verdaderamente importantes, entonces necesitamos pararnos y pensar sobre la forma correcta de actuar, principalmente, porque estamos lidiando con el sufrimiento o la felicidad de otras personas. Desde el momento en que J. Haidt y J. Greene rechazan este comportamiento racional respecto a la decisión moral, no están llevando a cabo una concepción adecuada de la moralidad. Los juicios morales racionales no son racionalizaciones pos hoc que enmascaran o justifican un impulso emocional, sino verdaderas deliberaciones que guían nuestra conducta. Ese pararse y pensar sobre lo moralmente correcto está también posibilitado por ciertos mecanismos neuronales.

Estas ideas de D. Pffaf sobre el papel jugado por el sufrimiento y el dolor están en consonancia con la concepción que E. G. Marcus elabora de la ansiedad, que al estar basada en el rol del sistema defensivo de vigilancia nos posibilita dejar de lado nuestros hábitos, dudar de ellos, criticarlos y pensar nuevas posibilidades. Ya afirmé que el rol del sistema de vigilancia es decirnos cuándo las condiciones que nos rodean favorecen o no nuestras tareas en curso. Al hacer esto, éste se convierte en un sistema de alerta que nos hace sentirnos más ansiosos cuando la incertidumbre de las consecuencias de nuestras acciones es mayor. Así, la confianza en los grupos a los que pertenecemos o los hábitos que nos conforman descienden drásticamente cuando la ansiedad aumenta, con ello nos volvemos más racionales y preparados para considerar diferentes alternativas. Sufrir ansiedad hace a la gente más abierta. Es muy fácil observar este efecto en el mundo de los hinchas; sólo tenemos que prestar atención a la reacción de la grada cuando un equipo lleva muchas derrotas consecutivas; los hinchas son muchos más críticos con la forma de jugar del equipo y también con las instituciones responsables de su fracaso.

Una vez que Donal Pfaff ha explicado el «camino negativo» de provocar esta disolución de fronteras que nos permite comportarnos de modo ético -imparcialidad-, va a tratar de explicitar una «vía positiva». Para ello se centrará en los mecanismos cerebrales encargados del placer, el amor y los actos de socialización, prestando especial atención al papel de dos hormonas, oxitocina y vassopresin, primariamente relacionadas con el cuidado maternal y paternal, pero que han acabado, como resultado de la evolución, influyendo en relaciones mucho más amplias como la amistad o relaciones sociales que nada tienen que ver con el parentesco o el sexo. ${ }^{34}$

Las relaciones de amistad y compañerismo que se establecen entre hinchas de un mismo equipo provocan la segregación de varios tipos de hormonas que, reduciendo sus niveles de ansiedad, hacen a los individuos más propensos a establecer relaciones con aquellos que consideran compañeros. Por ejemplo, la oxitocina nos liga a nuestros iguales y a los grupos que pertenecemos, sin embargo, como ya se explicó, parece hacernos más propensos a no considerar moralmente a aquellos que pertenecen a otros grupos diversos pues ponen en peligro nuestro status social. A juicio de Donald Pffaf, ese mismo efecto de ligazón por medio del cuidado y el cariño puede acabar trascendiendo los límites de las comunidades a las que pertenecemos.

\footnotetext{
${ }^{33}$ Ibíd., p. 76.

${ }^{34}$ Ibíd p. 117.
} 
Las fronteras de ese yo social del que formamos parte pueden incrementarse en cuanto que los sujetos perciben que, aún siendo hinchas de un equipo, siguen compartiendo muchos elementos con los rivales, por ejemplo, el amor a un deporte o, simplemente, una humanidad común. Así es cómo podrían explicarse, por elegir un caso particular, las muestras de solidaridad y unión entre hinchas de diversos equipos que acontecieron tras el paro cardiaco que sufrió el jugador francés Fabrice Muamba durante un partido de la F.A. Cup inglesa. En ese momento, las fronteras que separaban a las diversas hinchadas se diluyeron y éstos entendieron que, a pesar de todo, y más allá de los colores, somos principalmente personas que merecemos un igual reconocimiento y que, de hecho, estamos ligados y obligados a realizar tareas comunes que nos afectan a todos, independientemente de las comunidades particulares a las que pertenezcamos.

Así pues, para concluir, nada indica que, desde un punto de vista neuronal, los esfuerzos de una disciplina como la ética del deporte por expandir y realizar el ideal del fair play sean inservibles y utópicos. Es más, dados los dos contra-argumentos presentados, el fair play relativo al comportamiento de los hinchas deportivos no sólo es posible, sino que, además, concuerda con nuestra configuración neuronal.

\section{CONCLUSIÓN: EL FAIR PLAY ES POSIBLE}

Siguiendo las ideas y argumentos aportados hasta aquí, querría concluir diciendo que, aunque las siguientes exigencias de la neuroética pueden ser ciertas:

- Somos seres de hábitos.

- Nuestra configuración emotiva y neuronal es importante en nuestro comportamiento porque están a la base de nuestros hábitos.

- Los hinchas están dirigidos por fuertes lazos emocionales creados por la lógica interna del espectáculo deportivo.

Sin embargo, es también cierto que no estamos totalmente determinados por nuestros mecanismos emocionales y cerebrales sino que somos capaces de bloquear su influencia y fundamentar racionalmente nuestro comportamiento. Por lo tanto, los hinchas pueden comportarse de un modo racional y critico a pesar de los lazos emocionales que mantienen con su alrededor. Ya aporté dos argumentos a favor de esta idea, el primero, siguiendo la propuesta de Adela Cortina y, el segundo, podemos encontrar mecanismos neuronales que facilitan comportarnos apelando a la evaluación racional de nuestra conducta.

FrANCISCO JAVIER LÓPEz FríAs es Becario FPU en el Departamento de Filosofía del Derecho, Moral y Política de la Facultad de Filosofía y Ciencias de la Educación de la Universidad de Valencia.

Línea de investigación:

Tutorizado por Jesús Conill Sancho, prepara su tesis doctoral en ética del deporte. Más concretamente en las implicaciones que los métodos de dopaje pueden tener sobre la práctica deportiva, centrándose especialmente en aquellos métodos de dopaje derivados de lo que 
comienzan a llamarse como «técnicas de mejoramiento humano», es decir, intervenciones neuronales, genéticas, robóticas, etc.

\section{Publicaciones recientes:}

(2011) «Filosofía del deporte: Origen y desarrollo» en Dilemata: Revista Internacional de Éticas aplicadas, Volumen 5, pp. 1-19.

(2010) «Reivindicando una ética del deporte como filosofía aplicada: El deporte como cuestión moral» en Dilemata: Revista Internacional de Éticas aplicadas, Volumen 2, pp. 17-31.

Dirección electrónica: Francisco.Javier.Lopez@uv.es 